\title{
Common Input across Motor Nuclei Mediating Precision Grip in Humans
}

\author{
Gregory B. Hockensmith, Soren Y. Lowell, and Andrew J. Fuglevand \\ Department of Physiology, College of Medicine, University of Arizona, Tucson, Arizona 85721
}

\begin{abstract}
Short-term synchrony was measured for pairs of motor units located within and across muscles activated during a task that mimicked precision grip in the dominant and nondominant hands of human subjects. Surprisingly, synchrony for pairs of motor units residing in separate muscles (flexor pollicis longus, a thumb muscle, and flexor digitorum profundus, an index-finger muscle) was just as large as that for pairs of units both within the thumb muscle. Furthermore, the high level of synchrony seen across muscles in the dominant hand was absent in the nondominant hand. These results suggest that descending pathways diverge to provide extensive common input across motor nuclei involved in the precision grip and that such divergence might contribute to the preferred use of one hand over the other.
\end{abstract}

Key words: motoneuron; motor unit; corticospinal; synchrony; handedness; hand

\section{Introduction}

Virtually all movements require the coordinated action of multiple muscles. For example, numerous muscles of the forearm and hand are engaged in movements of individual digits or in the production of the precision grip in human and nonhuman primates (Maier and Hepp-Reymond, 1995; Schieber, 1995). How the nervous system orchestrates this activity is not fully understood. There is general agreement, however, that the ability to coordinate muscles during fractionated movements of the digits is in some way related to the prevalence of monosynaptic and disynaptic connections between the motor cortex and the motor neurons supplying the muscles of the hand (Heffner and Masterton, 1975; Bortoff and Strick, 1993; Porter and Lemon, 1993; Sasaki et al., 2004). Two nonmutually exclusive scenarios, therefore, can be envisioned as to how such pathways descending from the cerebral cortex might be organized to select specific combinations of muscles needed to perform particular voluntary tasks (Schieber, 1990; Bremner et al., 1991c; Hepp-Reymond et al., 1996). In one, selection is flexible and depends on the particular ensemble of cortical neurons engaged, which, in turn, activates specific muscles through separate descending pathways. In the other, selection of muscles into functional groups is determined by the pattern of divergence of descending fibers across spinal motor nuclei (Fetz and Cheney, 1980; Shinoda et al., 1981; Buys et al., 1986). This latter type of organization, although less flexible, might underlie the assemblage of muscles into synergistic groups needed to generate elemental movements that are the building blocks of the behavioral repertoire of an animal.

Manipulation of objects between the thumb and forefinger in the precision grip represents one of these fundamental motor

\footnotetext{
Received Jan. 5, 2005; revised March 29, 2005; accepted April 1, 2005.

This work was supported by National Institutes of Health Grant NS39489 (A.J.F.)

Correspondence should be addressed to Dr. Andrew J. Fuglevand, Department of Physiology, University of Arizona, P.0. Box 210093, Tucson, AZ 85721-0093. E-mail: fuglevan@u.arizona.edu. DOI:10.1523/JNEUROSCI.0046-05.2005

Copyright $\odot 2005$ Society for Neuroscience $\quad$ 0270-6474/05/254560-05\$15.00/0
}

behaviors that is characteristic of human and many nonhuman primates. The delicate control offered by the precision grip requires accurate matching of the normal forces exerted by the distal segments of the thumb and index finger (Forssberg et al., 1991). This implies that the muscles that flex the distal segments of the thumb [flexor pollicis longus (FPL)] and forefinger [digit 2 compartment of flexor digitorum profundus (FDP2)] must be tightly coordinated during the precision grip. Such coordination theoretically could be enacted by a descending pathway that diverges to supply both pools of motor neurons innervating the two muscles with common input.

Therefore, to gain insight into the means by which the CNS enlists muscles in the elaboration of stereotyped motor tasks, we evaluated the extent of common input to motor nuclei supplying FPL and FDP2 during a task that mimicked precision grip in both the dominant and nondominant hands of human subjects. The extent of common, last-order synaptic input was estimated from the level of short-term synchrony in the discharge times of pairs of motor units recorded from the two muscles (Sears and Stagg, 1976; Kirkwood and Sears, 1978). For comparison, synchrony was also assessed for pairs of motor units residing within the same muscle and for pairs of motor units situated in a different set of muscles used in a less common form of the precision grip.

\section{Materials and Methods}

Twenty-one experiments were performed on 10 healthy human volunteers (five females, five males) between the ages of 22 and 43 years. The Institutional Human Investigation Committee approved the procedures, and all subjects gave informed consent. All subjects were markedly righthand dominant with laterality quotients greater than +0.9 , as tested with the Edinburgh Handedness Inventory (Oldfield, 1971). Motor unit recordings were made from FPL and from FDP. FPL flexes the phalanges of digit 1 (thumb). FDP is a deep muscle residing in the ventral forearm that gives rise distally to four tendons that insert on the anterior surface of the distal phalanges of digits $2-5$ (index-little fingers). FDP can cause flexion at any of the joints over which it passes (wrist, metacarpalphalangeal joints, interphalangeal joints) and is the only muscle that flexes the distal 

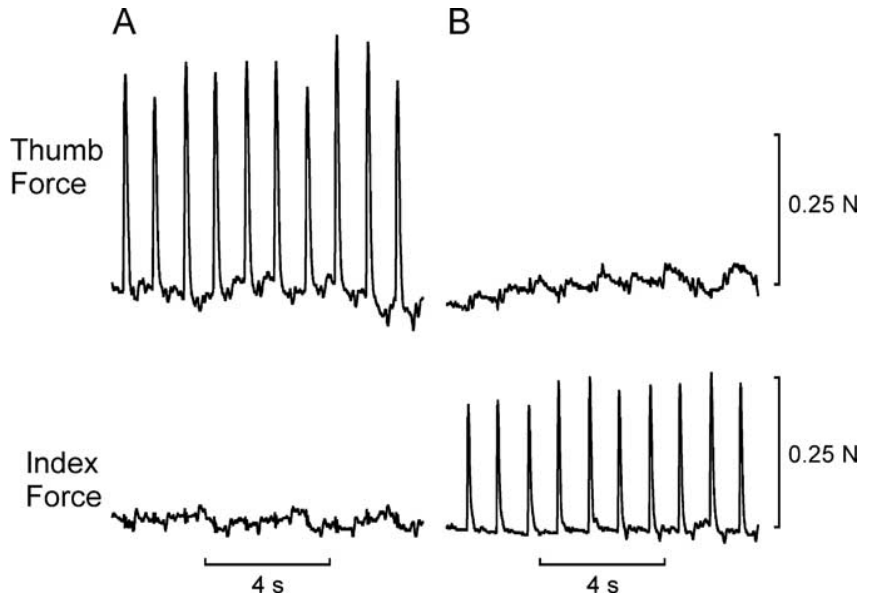

Figure 1. Example isometric force responses recorded from the distal segments of the thumb and index finger to stimulation $(2.5 \mathrm{~mA}, 0.5 \mathrm{~ms}$ duration, $1 \mathrm{~Hz})$ of FPL $(\boldsymbol{A})$ and FDP2 $(\boldsymbol{B})$.

interphalangeal joints. In the present experiments, we sampled motor unit activity only from the divisions of FDP that insert on digit 2 or digit 3 .

Subjects sat in a dental chair with their forearm supported on an adjustable platform. The upper arm was slightly abducted, and the elbow flexed at an angle of $\sim 150^{\circ}$. The hand was supinated $\sim 60^{\circ}$ from vertical and secured in this position with a Velcro strap at the wrist and with foam-padded magnetic stands buttressing either side of the hand. Leather cuffs encircled the volar surface of the distal segments of the thumb and index (or middle) finger and were attached to two isometric force transducers with lightweight cables. A modest extensor preload $(\sim 1.5 \mathrm{~N})$ was applied to both cables, which held the digits apart by a distance of $\sim 12 \mathrm{~cm}$.

Motor unit recording. Motor unit action potentials were recorded with two high-impedance tungsten microelectrodes inserted percutaneously from the ventral surface of the forearm and into FPL and FDP. Surface electrodes attached to the skin overlying the radius served as reference electrodes for the two intramuscular electrodes. Weak electrical stimulation was used initially to verify microelectrode placement in target compartments of FDP or in FPL based on the forces evoked on each digit (Fig. 1). In addition, the presence of evoked flexion of the distal segment of digit 2 or 3 was used to verify placement in FDP as opposed to another digit flexor, the flexor digitorum superficialis. After electrical stimulation, electrodes were connected to differential amplifiers, and the intramuscular electromyographic (EMG) signals were amplified (1000), bandpass filtered $(0.3-3 \mathrm{kHz})$, displayed on oscilloscopes, and routed to an audio amplifier.

Protocol. Subjects were instructed to squeeze gently the thumb and index finger together against the leather cuffs connected to the force transducers to mimic grasping of an object. The microelectrodes were manipulated during this task until action potentials of motor units could be clearly identified on each electrode. Once motor units were identified on the two electrodes, subjects were instructed to keep the units discharging at low, steady rates for $\sim 300 \mathrm{~s}$ using audio and visual feedback while flexing the distal segments of the index finger and thumb. The digits were not constrained to exert the same level of force during the task. During the trials, force and intramuscular EMG signals were digitally sampled at 2 and $20 \mathrm{kHz}$, respectively, using the Spike2 data acquisition and analysis system (Cambridge Electronics Design, Cambridge, UK). On occasion, trials were aborted when the activity of a unit was lost (presumably as a result of electrode movement) or when excessive activity in other detected units made reliable discrimination unlikely. Subjects received 1-2 min of rest between trials. After each recording, the positions of both microelectrodes were adjusted until the action potentials of a presumed different pair of motor units could be identified. Electrical stimulation was performed between each trial to ensure that the electrodes remained within the target muscles.

In some trials, the procedures were identical to those described above, except that both electrodes were placed in the thumb muscle (FPL). This was done to obtain comparative estimates of common input to pairs of motor units that reside within a single muscle. In other trials, motor unit activity was recorded from FDP3 and from FPL during a task identical to that described above, except that it involved sustained flexion of the distal segments of the middle finger and thumb. These data were collected to examine the extent of common input across motor nuclei associated with what might be a less common form of the precision grip. And finally, in a separate series of experiments, motor unit data were recorded from FDP2 and FPL of the left (nondominant) hand during flexion of the left thumb and index finger. This was done to ascertain whether or not the pattern of common input across motor nuclei was associated with handedness.

Data analysis. All trials in which the activity of the units could be reliably discriminated for at least 2 min were included in the analysis. Data were analyzed according to methods described in detail recently (Keen and Fuglevand, 2004). Briefly, motor unit discrimination was performed off-line using a template-matching algorithm based on waveform shape and amplitude (Spike2; Cambridge Electronics Design). Cross-correlation histograms ( $1 \mathrm{~ms}$ bins, $\pm 100 \mathrm{~ms}$ ) were then constructed from the discharge times of the discriminated motor units detected on the separate electrodes. A narrow peak in the histogram around time 0 represents synchronized firing of the two units. The magnitude of this synchronous peak is thought to indicate of the degree of common, last-order inputs to the two neurons (Sears and Stagg, 1976; Kirkwood and Sears, 1978; Kirkwood, 1979).

Peaks in the histograms were identified using the cumulative sum (cusum) procedure, which involves progressively summing the differences in the number of counts in each bin of the cross-correlogram from the mean bin count (Ellaway, 1978). The baseline mean was calculated as the mean count in the first and last $60 \mathrm{~ms}$ of the cross-correlogram. The peak boundaries in cross-correlograms were determined as the bins corresponding to 10 and $90 \%$ of the maximum rise in the cusum near time 0 (Schmied et al., 1993). The magnitude of peaks in cross-correlograms were quantified as the total number of counts within the boundaries of the peak above the baseline mean divided by the duration of the recording (Nordstrom et al., 1992). This synchronization index, referred to as common input strength (CIS), denotes the frequency of synchronous spikes for a pair of units in excess of those expected as a result of chance (i.e., extra synchronous spikes per second).

When cross-correlograms do not exhibit clear peaks, the method described above for identifying the region of the histogram for calculation of CIS is not reliable. Therefore, for cases of nonsignificant peaks in the cross-correlogram, CIS was automatically calculated for an $11 \mathrm{~ms}$ region of the cross-correlogram centered at time 0 (Semmler and Nordstrom, 1995). The significance of the cross-correlogram peak was determined according to the method described by Schmied et al. (1993), in which the average number of counts in the peak region must be $>3$ SDs above the mean count $(z$ score $\geq 1.96$ ) to be considered significant.

A one-way ANOVA was used to determine whether mean CIS values were significantly different for each of three combinations of paired motor unit recordings from the dominant hand: both units within the FPL ("thumb-thumb"), one unit in FPL and one unit in FDP2 ("thumbindex"), and one unit in FPL and one unit in FDP3 ("thumb-middle"). Tukey's post hoc analysis was used to identify differences in CIS across motor-unit combinations. In addition, a two-tailed Student's $t$ test was performed to determine whether CIS values were different for thumbindex pairings of units between the dominant and nondominant hands. Values are reported as means $\pm \mathrm{SD}$ with a probability of 0.05 selected as the level of statistical significance.

\section{Results}

A total of 181 motor units were recorded from 10 subjects during weak voluntary flexion of the distal segments of the thumb and index finger or thumb and middle finger. Because motor unit recordings were made only during low-force contractions, the motor units sampled in this study were restricted only to lowthreshold, presumably weak motor-unit types. The average dis- 
charge rate for all units was $9.8 \pm 1.3 \mathrm{~Hz}$, and the average number of spikes per unit used in the analysis was $2273 \pm 866$. From the discharge times of units detected on separate electrodes, 107 cross-correlograms were constructed. In some trials, more than one unit was recorded on an electrode, allowing for more than one crosscorrelation per trial. Twenty-six of the cross-correlograms were for pairs of units both within FPL (thumb-thumb), 25 were for FPL-FDP2 pairs (thumb-index), 25 were for FPL-FDP3 pairs (thumb-middle), and 31 were for FPL-FDP2 pairs (thumb-index) recorded in the nondominant hand.

Figure $2 \mathrm{~A}$ shows example crosscorrelograms for three different combinations of motor unit pairs recorded in the dominant hand. As has been shown previously (Datta and Stephens, 1990; Nordstrom et al., 1992), a prominent central peak indicating a relatively high level of synchrony was seen in the crosscorrelogram for the pair of motor units residing within the same hand muscle (Fig. 2A, thumb-thumb). Surprisingly, the magnitude of synchrony for the pair of motor units residing in different muscles (FPL-FDP2) (Fig. $2 A$, thumb-index) was virtually the same as that for the pair of motor units within the same muscle. Synchrony, however, was much less for the FPL-FDP3 pair of units (Fig. $2 \mathrm{~A}$, thumbmiddle). Figure $2 B$ shows the mean $\pm \mathrm{SD}$ CIS values for the three combinations of all motor units pairs recorded from the dominant hand. There was no significant difference in CIS between the thumbthumb and thumb-index combinations of motor unit pairs, although both of these were greater $(p<0.001)$ than the thumbmiddle combination. A large proportion of the cross-correlogram peaks were significant (see Materials and Methods) for both thumb-thumb (85\%) and thumb-index pairs (72\%), whereas only $16 \%$ were significant for the thumb-middle pairs.

Interestingly, however, these effects were seen only in the dominant (right) hand. Figure $3 \mathrm{~A}$ shows example crosscorrelograms for a thumb-index pair of units in the dominant hand and for a thumb-index pair in the nondominant hand. Little of the marked synchrony characterizing thumb-index pairs of the dominant hand was evident in the nondominant hand. Indeed, only $23 \%$ of the thumb-index motor unit pairs recorded in the nondominant hand exhibited significant crosscorrelogram peaks. The mean \pm SD CIS for all 31 of these pairs (Fig. $3 B$ ) was $0.12 \pm 0.09$ extra synchronous spikes/s, which was substantially and significantly $(p<0.001)$ lower than that for the 25 thumb-index pairs recorded from the dominant hand $(0.42 \pm$ $0.30)$.

Finally, the durations of the correlogram peaks (i.e., the $10-$ $90 \%$ rise time in the cusum) were relatively brief for all muscle combinations and were not significantly different from one another $(8.6 \pm 2.3,9.4 \pm 3.4,6.8 \pm 1.9$, and $8.4 \pm 4.2 \mathrm{~ms}$ for the dominant thumb-thumb, thumb-index, thumb-middle, and nondominant thumb-index combinations, respectively).

\section{Discussion}

We used cross-correlation analysis of the discharge times of motor units located in different muscles to estimate the extent to which their coordinated activity might be attributable to divergence of descending pathways providing common input across motor nuclei activated during the precision grip. We found a high level of synchrony among pairs of motor units residing in separate muscles critical to the performance of the precision grip (FPL and FDP2) that was indistinguishable from degree of synchrony found for motor units within the FPL. Furthermore, this effect was seen in the dominant but not in the nondominant hand. These results imply that matching of contact forces between the thumb and index finger in the dominant hand during the precision grip might be facilitated by descending pathways that diverge to operate on the two motor nuclei as a unit and thereby compel the two muscles to operate in unison.

The demonstration of common input across motor nuclei, however, does not by itself imply that the recipient nuclei are obligatorily linked together. Rather, it seems likely that both divergent and independent pathways supplying separate motor nuclei coexist and that the predominant set of pathways engaged 


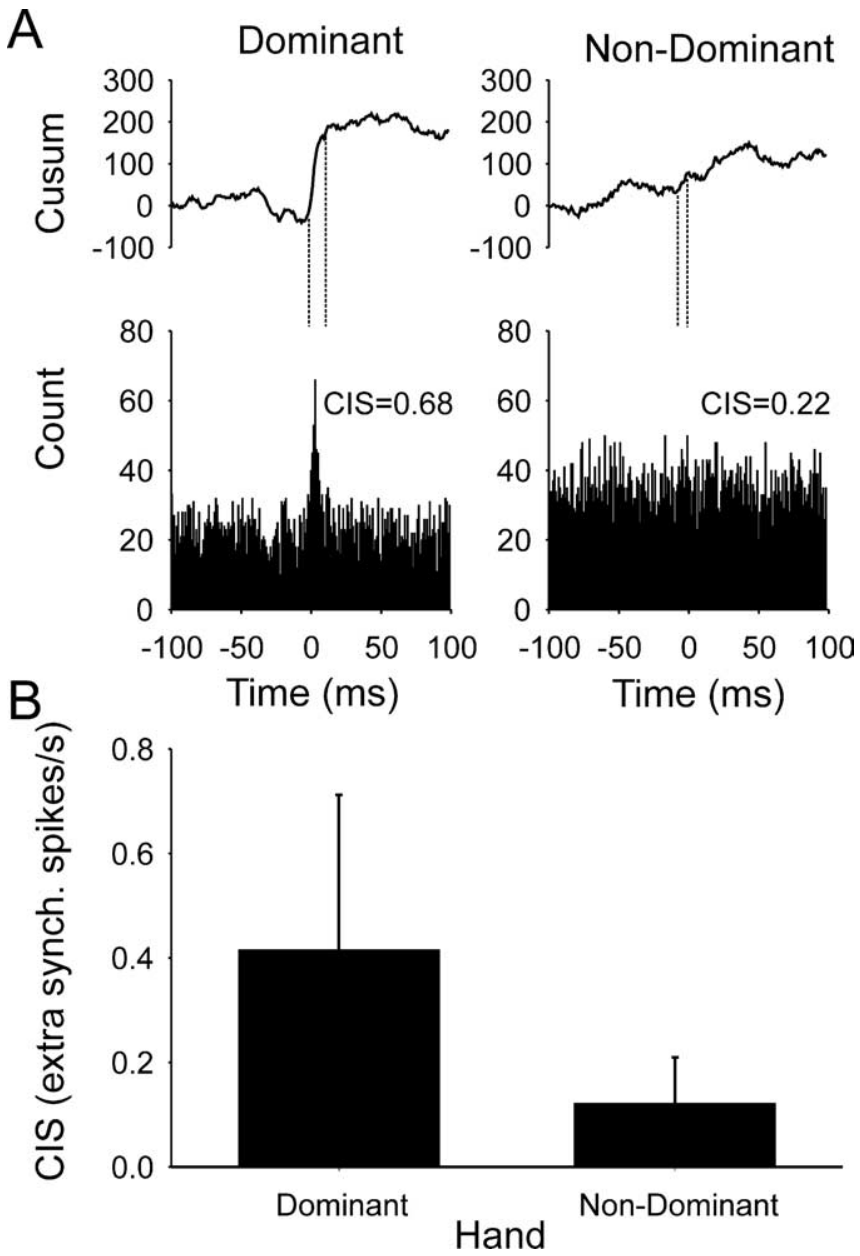

Figure 3. CIS for thumb-index pairs of units only located in dominant and nondominant hands. $\boldsymbol{A}$, Example cross-correlograms. $\boldsymbol{B}$, Mean (SD) CIS for all thumb-index pairs in the dominant and in the nondominant hand. synch., Synchronous.

depends on the contingencies of the task to be performed. Indeed, Bremner et al. (1991c) showed that the degree of synchrony across muscles of the hand can be modulated as a function of task.

\section{Limitations}

In the present experiments, rather than actually grasping an object with a precision grip, subjects simulated precision grip using an apparatus that allowed for independent recording of the force exerted by each digit. This was done to establish, using electrical stimulation, the muscular location of the electrodes (Fig. 1). This was a critical feature of our experimental design because of the relatively small sizes of the muscles and muscle compartments involved and their proximity to one another, which make accurate electrode placement difficult. Nevertheless, we do not believe the use of a simulated precision grip necessarily affected our results. Indeed, Winges and Santello (2004) recently reported a high level of FPL-FDP2 motor-unit synchrony (mean CIS, 0.49) in the right hand of human subjects during a task involving actual gripping of an object that was similar to the synchrony we found (mean CIS, 0.42) in our simulated gripping task.

\section{Inferences about common input based on synchrony}

Although it is not possible to assess directly the pattern of divergence of synaptic inputs across motor nuclei in the human spinal cord, information about the distribution of inputs to motor neu- rons can be estimated from the relationship in the timing of discharges between motor units (Sears and Stagg, 1976; Kirkwood, 1979). Theoretically, the existence of last-order presynaptic fibers that diverge to provide common excitatory input should provoke near simultaneous excitatory potentials in the recipient neurons and thereby slightly increase the probability that some of the neurons will be brought to action-potential threshold at the same time (Perkel et al., 1967; Kirkwood and Sears, 1978). Consequently, when the timing of action potentials is compared among neurons that receive such common input, there is a significant coincidence in their discharge, revealed as a narrow peak near time 0 in the cross-correlogram constructed from the relative firing times of two simultaneously active units. Centrally located peaks in cross-correlograms can also arise because presynaptic neurons themselves are synchronized to some degree, but these tend to have a broader duration (more than $\sim 10 \mathrm{~ms}$ ) than those arising directly from branched last-order inputs (Kirkwood et al., 1982). Although the peak widths for all combinations of units tested here averaged $<10 \mathrm{~ms}$, the possible contribution of separate although synchronized presynaptic inputs cannot be discounted. Nevertheless, the magnitude of the synchronous peak provides a noisy representation of the proportion of common (including synchronized presynaptic inputs) to noncommon inputs to the two neurons (Kirkwood and Sears, 1978). Because short-term synchrony appears to be minimally affected by lesions of the dorsal roots (Kirkwood et al., 1982) yet is markedly diminished with section of descending pathways (Kirkwood et al., 1982; Datta et al., 1991), the source of such synchrony is thought to be primarily central rather than peripheral.

\section{Within- and across-muscle synchrony}

Substantial synchrony has been shown previously to exist for most pairs of motor units within intrinsic (Datta and Stephens, 1990; Bremner et al., 1991a; Nordstrom et al., 1992) and within extrinsic hand muscles (Bremner et al., 1991b; Schmied et al., 1993; Keen and Fuglevand, 2004). These findings support the idea that presynaptic fibers branch extensively to provide broadly distributed input across motor neurons comprising a single motor nucleus (Mantel and Lemon, 1987). Fewer studies, however, have examined synchrony for pairs of motor units residing in different muscles. The limited data available indicate that synchrony is absent for certain combinations of muscles, for example, between extensor carpi radialis and extensor digitorum (Gibbs et al., 1995). For other sets of muscle combinations, synchrony has been shown to exist but always to a lesser extent than for motor units residing within the same muscle (Bremner et al., 1991a,b; Gibbs et al., 1995). The muscle combinations examined thus far that exhibit significant synchrony usually involve neighboring muscles or muscle compartments (Bremner et al., 1991b, Keen and Fuglevand, 2004; Reilly et al., 2004) (cf. Winges and Santello, 2004).

What is novel about the present findings is that the magnitude of across-muscle synchrony for FPL-FDP2 was practically as large as that found within individual hand muscles. Some degree of synchrony might be expected between FPL and FDP2 because of their physical proximity (and presumed contiguity of their spinal motor nuclei) to one another. Indeed, the level of synchrony between the more spatially separated FPL-FDP3 muscles was much lower than that for the neighboring FPL-FDP2 combination. However, simple "spill-over" of descending projections across adjacent motor nuclei would not seem to account for the full extent of synchrony seen between FPL and FDP2. This is because synchrony was virtually absent between the nondomi- 
nant FPL and FDP2, the motor nuclei of which presumably reside as near to one another as do the motor nuclei supplying the dominant side. It seems possible, therefore, that extensive divergence of descending inputs across motor nuclei participating in the production of the precision grip in the dominant hand represents an anatomical strategy that serves to tightly couple the activities of key muscles involved in that task.

\section{Synchrony and handedness}

Previous studies that have compared the extent of motor unit synchrony within muscles of the dominant and nondominant arms have yielded mixed results. For example, Schmied et al. (1994) found greater motor unit synchrony in extensor carpi radialis on the dominant side for both right-handed and lefthanded subjects, whereas Semmler and Nordstrom (1995) found greater synchrony in first dorsal interosseus on the nondominant side of right-handed subjects and no difference in synchrony across hands of left-handed subjects. To our knowledge, motor unit synchrony across muscles in the dominant and nondominant arms has not been examined previously. In the present study, we found that the magnitude of synchrony for FPL-FDP2 pairs of motor units was markedly higher in the dominant hand compared with the nondominant hand. However, we cannot be certain that such differences actually relate to hand dominance because we did not test left-handed subjects. Indeed, certain features of the pyramidal tract projecting to the right side of the spinal cord tend to be different from that projecting to the left, regardless of handedness (Kertesz and Geschwind, 1971; Nathan et al., 1990). Nevertheless, the possibility that lateralized differences in the extent of descending-path divergence across motor nuclei involved in the precision grip might contribute to the preferred use of one hand over the other is an intriguing prospect that warrants additional investigation.

\section{References}

Bortoff GA, Strick PL (1993) Corticospinal terminations in two new-world primates: further evidence that corticomotoneuronal connections provide part of the neural substrate for manual dexterity. J Neurosci 13:5105-5118.

Bremner FD, Baker JR, Stephens JA (1991a) Correlation between the discharges of motor units recorded from the same and from different finger muscles in man. J Physiol (Lond) 432:355-380.

Bremner FD, Baker JR, Stephens JA (1991b) Variation in the degree of synchronization exhibited by motor units lying in different finger muscles in man. J Physiol (Lond) 432:381-399.

Bremner FD, Baker JR, Stephens JA (1991c) Effect of task on the degree of synchronization of intrinsic hand muscle motor units in man. J Neurophysiol 66:2072-2083.

Buys EJ, Lemon RN, Mantel GWH, Muir RB (1986) Selective facilitation of different hand muscles by single corticospinal neurones in the conscious monkey. J Physiol (Lond) 381:529-549.

Datta AK, Stephens JA (1990) Synchronization of motor unit activity during voluntary contraction in man. J Physiol (Lond) 422:397-419.

Datta AK, Farmer SF, Stephens JA (1991) Central nervous pathways underlying synchronization of human motor unit firing studied during voluntary contractions. J Physiol (Lond) 432:401-425.

Ellaway PH (1978) Cumulative sum technique and its application to the analysis of peristimulus time histograms. Electroencephalogr Clin Neurophysiol 45:302-304.

Fetz EE, Cheney PD (1980) Postspike facilitation of forelimb muscle activity by primate corticomotoneuronal cells. J Neurophysiol 44:751-772.

Forssberg H, Eliasson AC, Kinoshita H, Johansson RS, Westling G (1991)
Development of human precision grip I: basic coordination of force. Exp Brain Res 85:451-457.

Gibbs J, Harrison LM, Stephens JA (1995) Organization of inputs to motoneurone pools in man. J Physiol (Lond) 485:245-256.

Heffner RS, Masterton RB (1975) Variation in form of the pyramidal tract and its relationship to digital dexterity. Brain Behav Evol 12:161-200.

Hepp-Reymond MC, Huesler EJ, Maier MA (1996) Precision grip in humans. Temporal and spatial synergies. In: Hand and brain: the neurophysiology and psychology of hand movements (Wing AM, Haggard P, Flanagan JR, eds), pp 37-68. San Diego: Academic.

Keen DA, Fuglevand AJ (2004) Common input to motor neurons innervating the same and different compartments of the human extensor digitorum muscle. J Neurophysiol 91:57-62.

Kertesz A, Geschwind N (1971) Patterns of pyramidal decussation and their relationship to handedness. Arch Neurol 24:326-332.

Kirkwood PA (1979) On the use and interpretation of cross-correlation measurements in the mammalian central nervous system. J Neurosci Methods 1:107-132.

Kirkwood PA, Sears TA (1978) The synaptic connexions to intercostal motoneurones as revealed by the average common excitation potential. J Physiol (Lond) 275:103-134.

Kirkwood PA, Sears TA, Tuck DL, Westgaard RH (1982) Variations in the time course of the synchronization of intercostal motoneurones in the cat. J Physiol (Lond) 327:105-135.

Maier MA, Hepp-Reymond MC (1995) EMG activation patterns during force production in precision grip. I. Contribution of 15 finger muscles to isometric force. Exp Brain Res 103:108-122.

Mantel GWH, Lemon RN (1987) Cross-correlation reveals facilitation of single motor units in thenar muscles by single corticospinal neurones in the conscious monkey. Neurosci Lett 77:113-118.

Nathan PW, Smith MC, Deacon P (1990) The corticospinal tracts in man: course and location of fibres at different segmental levels. Brain 113:303-324.

Nordstrom MA, Fuglevand AJ, Enoka RM (1992) Estimating the strength of common input to human motoneurons from the cross-correlogram. J Physiol (Lond) 453:547-574.

Oldfield RC (1971) The assessment and analysis of handedness: the Edinburgh inventory. Neuropsychologia 9:97-113.

Perkel DH, Gerstein GL, Moore GP (1967) Neuronal spike trains and stochastic point processes. Biophys J 7:419-440.

Porter R, Lemon RN (1993) Corticospinal function and voluntary movement. New York: Oxford UP.

Reilly KT, Nordstorm MA, Schieber MH (2004) Short-term synchronization between motor units in different functional subdivisions of the human flexor digitorum profundus muscle. J Neurophysiol 92:734-742.

Sasaki S, Isa T, Pettersson LG, Alstermark B, Naito K, Yoshimura K, Seki K, Ohki Y (2004) Dexterous finger movements in primate without monosynaptic corticomotoneuronal excitation. J Neurophysiol 92:3142-3147.

Schieber MH (1990) How might the motor cortex individuate movements? Trends Neurosci 13:440-445.

Schieber MH (1995) Muscular production of individuated finger movements: the roles of extrinsic finger muscles. J Neurosci 15:284-297.

Schmied A, Ivarsson C, Fetz EE (1993) Short-term synchronization of motor units in human extensor digitorum communis muscle: relation to contractile properties and voluntary control. Exp Brain Res 97:159-172.

Schmied A, Vedel JP, Pagni S (1994) Human spinal lateralization assessed from motoneurone synchronization: dependence on handedness and motor unit type. J Physiol (Lond) 480:369-387.

Sears TA, Stagg D (1976) Short-term synchronization of intercostal motorneuron activity. J Physiol (Lond) 263:357-381.

Semmler JG, Nordstrom MA (1995) Influence of handedness on motor unit discharge properties and force tremor. Exp Brain Res 104:115-125.

Shinoda Y, Yokota JI, Futami T (1981) Divergent projection of individual corticospinal axons to motoneurons of multiple muscles in the monkey. Neurosci Lett 23:7-12.

Winges SA, Santello M (2004) Common input to motor units of digit flexors during multi-digit grasping. J Neurophysiol 92:3210-3220. 